Mohsen S. Sajadieh, Anders Thorstenson \& Mohammad R. Akbari Jokar

Comparing sourcing strategies in two-echelon supply chains

CORAL - Centre for Operations

Research Applications in Logistics 


\title{
Comparing sourcing strategies in two-echelon supply chains
}

\author{
Mohsen S. Sajadieh ${ }^{\mathrm{a}}$, Anders Thorstenson ${ }^{\mathrm{b}, *}$, Mohammad R. Akbari Jokar $^{\mathrm{a}}$ \\ ${ }^{a}$ Department of Industrial Engineering, Sharif University of Technology, Tehran, Iran \\ ${ }^{b}$ CORAL - Centre for O.R. Applications in Logistics, Department of Business Studies, \\ Aarhus School of Business, Aarhus University, Denmark
}

\begin{abstract}
In this paper we investigate four sourcing models with respect to both cooperative or noncooperative planning strategies, and sole or dual sourcing. A two-stage supply chain is considered, which includes a single buyer and either a single or dual vendor(s). At the buyer the product is consumed at a constant rate, and an $(r, Q)$ policy is used to generate replenishment orders. The vendors have a finite production rate and ship to the buyer in equal-sized batches. The delivery lead time is stochastic. Shortages are allowed at the buyer's side and are fully backordered. The cost function comprises five elements: inventory holding costs at the buyer and the vendors, backorder costs and ordering costs at the buyer, and setup costs at the vendors. The objective is to minimize the total system costs incurred by the buyer and the vendors. As there is no dominating combined strategy, a major finding is that determining the best strategy requires a detailed analysis. Furthermore, when total system costs are taken into account, dual sourcing does not appear as beneficial as sometimes claimed in the literature on lot splitting.
\end{abstract}

Keywords: Coordinated vendor-buyer model, dual sourcing, integrated production-inventory system, joint economic lot sizing, order splitting.

\footnotetext{
* Corresponding author; e-mail: ath@asb.dk, tel.: +4589486318

Mailing address: CORAL, Aarhus School of Business, Aarhus University, Fuglesangs Allé 4, DK-8210 Aarhus, Denmark
} 


\section{Introduction}

In today's highly competitive markets the establishment of a good sourcing strategy is a prerequisite for effective supply chains. As sourcing becomes a fundamental element in supply chain management, organizations are considering alternatives to traditional sourcing principles. The best sourcing strategy based on the internal and external factors at hand may be defined with respect to several different attributes and among these we find the number of suppliers and the level of coordination with the suppliers. This paper analyzes the cost implications of these two strategic choices and their combined effects.

The choice of the number of suppliers is an issue that has been under much discussion in the literature on different management concepts. There are several arguments for having either a single supplier or having multiple suppliers. Concepts such as Just-In-Time, Lean Manufacturing, and Total Quality Management often encompass the suggestion to reduce the supplier base and to build long-term relationships with important suppliers. During the past decade trends in supplier management and sourcing also show increased global sourcing, reductions of the supplier bases, and developments of long-term relationships with strategic suppliers. However, other developments such as increased risk exposure due to globalization and the availability of e-commerce with opportunities for spot market replenishments have emphasized the advantages of multiple sourcing. With respect to risk exposure splitting of replenishment orders among two or more suppliers has been suggested as a mechanism for risk hedging in supply chain management. In this paper we study order splitting and its relation to replenishment costs.

The coordination of activities within a supply chain facilitated by the sharing of information allows companies to provide products and services at a reduced cost. In traditional inventory management, production and shipment policies for a manufacturer and a buyer in a dyadic supply chain link have been managed independently. As a result, the 
optimal lot size for the buyer may not result in an optimal policy for the vendor and vice versa. To overcome this difficulty, the integrated vendor-buyer model has been developed, where the joint total relevant costs for the buyer and the vendor are minimized. The structure of global supply chains can be very complex. Nevertheless, link-by-link understanding of joint optimal policies can provide useful insights. In the literature, one stream of research that deals with this integrated vendor-buyer problem is referred to as the joint economic lot sizing (JELS) problem. This paper is related to this stream of research.

Goyal (1976) was one of the first to introduce the idea of a joint total cost for a single vendor and a single buyer scenario under the assumption of an infinite production rate for the vendor and a lot-for-lot policy for the shipments from the vendor to the buyer. Banerjee (1986) relaxed the infinite production rate assumption. Later, Goyal (1988) contributed to the efforts of generalizing the problem by relaxing the assumption of a lot-for-lot shipment policy. He assumed that the production lot is shipped in a number of equal-size shipments.

Goyal (1995) then developed a model where the shipment size increases by a factor equal to the ratio of the production rate to the demand rate. He formulated the problem and developed an expression for the first shipment size as a function of the number of shipments. Hill (1997) generalized the model of Goyal (1995) by including the geometric growth factor as a decision variable. He suggested a solution method based on an exhaustive search for both the growth factor and the number of shipments in certain ranges. Hill (1999) relaxed the assumptions of the shipment policy and developed an optimal solution for the problem. He showed that the structure of the optimal policy includes shipments increasing in size according to a geometric series followed by equal-sized shipments.

The basic JELS models have been extended in many different directions. Broadly speaking, the existing literature on JELS can be divided into different categories according to characteristics such as "quality", "controllable lead time", "multiple buyers", "setup and 
order cost reduction", "transportation", "deteriorating items", "three-level supply chains", etc. (see e.g. Huang (2004), Yang and Wee (2002), Muson and Rosenblatt (2001), and Toptal et al. (2003)). It is beyond the scope of this paper to discuss these contributions in detail. Instead, we refer to Ben-Daya et al. (2008) for a comprehensive review of the JELS problems.

With regard to multiple sourcing, the order-splitting literature focuses on splitting orders simultaneously among several suppliers in order to obtain reductions in the total system cost. The first paper in this stream of literature was published by Sculli and Wu (1981). They studied an inventory system with two sources, where lead times are normally distributed. The numerical results indicate that the reorder level for a given stock-out probability is lower than in the single sourcing system. Sculli and Shum (1990) extended this model to more than two sources. Ramasesh et al. (1991) developed models with two sources where demand is assumed to be constant and both sources have identical lead-time distributions. Ramasesh et al. (1993) later extended their work by relaxing the assumptions of identical lead-time distributions, unit supply prices, and split proportions for the two sources.

Lau and Zhao (1993) considered a model with stochastic demand and lead times. They developed a procedure to identify the second best source. Chiang and Benton (1994) investigated a model with normally distributed demand and shifted exponential lead times. They showed that dual sourcing performs better than sole sourcing except in the cases where the ordering cost is high, the lead-time variability is low, or the customer service level is low. Hill (1996) used a similar framework for analyzing cycle stock under order splitting and showed that multiple sourcing reduces the average stock levels for any reasonable lead-time distribution.

Later, Sedarage et al. (1999) developed models of multiple sourcing in single-item inventory systems in which both lead times and demand are stochastic. Lead times may have 
different distributions and suppliers' purchasing prices may also be different. Ryu and Lee (2003) considered dual-sourcing models assuming exponentially distributed lead times and constant demand. They use expediting cost functions and demonstrate how an investment to reduce lead times can result in significant savings. For further literature we refer to the papers by Minner (2003) and Thomas and Tyworth (2006) which provide comprehensive surveys of order-splitting problems. The latter also includes a critical review of the order splitting literature. One of the key issues in their critique is the lack of analyses in a supply chain context. This issue is addressed in this paper.

The main contribution of this paper is the integration of the two above mentioned streams of literature in models which analyze four different sourcing scenarios. The models developed can be considered as JELS models in which more than one vendor/supplier manufactures the products. At the same time, they can be considered as order-splitting models in which the joint costs of buyer and suppliers/vendors are optimized. Thus, we analyze the combination of sourcing and coordination decisions to find the optimal strategy for different cases.

The best overall sourcing strategy is dependent upon a number of factors such as vendor capabilities, production stability, the level of trust among the supply chain parties, etc. However, in this paper we focus on the operational costs of setups, ordering, inventory holding, and shortages to determine the best sourcing option. When comparing these operational costs in four different sourcing scenarios, our results suggest that companies should focus primarily on the strategy of coordinating with a single vendor. Contrary to the results often found in the order-splitting literature, dual sourcing is not found to be advantageous for a wide range of parameters when total supply chain costs are considered.

The rest of the paper is organized as follows. In Section 2, the problems and the strategy scenarios are defined. Notations and assumptions are also introduced. Section 3 provides a 
specification of each of the sourcing models. The solution approaches developed are also discussed in this section. Section 4 presents numerical examples and an extensive sensitivity analysis. In Section 5, the two dual sourcing scenarios are extended to the case in which the suppliers are not necessarily identical. Numerical analyses are also presented in this section. Finally, the main findings and further research directions are summarized in Section 6.

\section{Problem definition and notations}

Consider the supply chain dyad for a product consisting of a single vendor and a single buyer. The final demand for the product is assumed to be deterministic and constant. The lead time on the other hand is stochastic. The lots delivered from the vendor to the buyer are equally sized batches. As soon as the inventory position at the buyer drops to $r$, an order of size $Q$ is issued by the buyer. The vendor manufactures the product at production rate $P$ and in lot sizes which are a multiple $n$ of $Q$. The cost of the system includes setup, ordering, holding, and shortage costs. The objective is to determine the number of lot shipments $n$, the reorder point $r$, as well as the order lot size $Q$ so that the total expected long-run average costs of vendor and buyer are minimized.

Considering sole and dual-sourcing strategies as well as cooperative and non-cooperative strategies, there is a total of four possible sourcing scenarios. In the cooperative strategy, the total expected costs are optimized jointly, whereas in the non-cooperative strategy, they are optimized individually. As can be seen in Figure 1, Scenario 1 represents the setting in which the buyer sources the product from a single vendor, and there is no cooperation between them regarding coordination of replenishment policies. Scenario 2 also represents the noncooperative case, but under a dual sourcing strategy. If the supply chain members decide to coordinate replenishments with each other, then Scenarios 1 and 2 transform into Scenarios 3 and 4 , respectively. 
Scenarios 1 and 3 have been studied before by Sajadieh et al. (2008). On the one hand, this paper can be considered an extension of their JELS model into the dual-sourcing strategy comparing all possible scenarios. On the other hand, it can also be viewed as an extension of the order-splitting models into an integrated model in which the total costs of the buyer and the vendors are optimized jointly. This contrasts with most previous studies of order splitting in the literature.

\section{Figure 1}

The following assumptions are common to all four scenarios:

1. The model deals with a single buyer for a single product.

2. The final customer demand rate $D$ is deterministic and constant.

3. Inventory is continuously reviewed and infinitely divisible. The buyer orders a lot of size $Q$ when the inventory position reaches the reorder point $r$.

4. Lead-time to replenish the buyer's order is a stochastic variable $L$ that follows an exponential distribution, i.e., $L \sim \exp (\lambda)$, with $E(L)=1 / \lambda$.

5. Replenishment orders do not cross in time. This is approximated by requiring that the probability of order crossover is small.

6. Shortages are allowed and completely backordered.

7. The vendor (manufacturer) has a finite production rate $P$ which is greater than the demand rate.

8. The vendor manufactures a batch $n Q$, where $n$ is an integer, at each set up, and each batch is delivered to the buyer in $n$ equally sized shipments.

9. The time horizon is infinite. 
The cost parameters are:

$A_{V} \quad$ Setup cost for the vendor(s)

$A_{B} \quad$ Ordering cost for the buyer

$h_{V} \quad$ Inventory holding cost for the vendor(s) per unit per unit time

$h_{B} \quad$ Inventory holding cost for the buyer per unit per unit time

$\pi \quad$ Backorder cost for the buyer per unit per unit time

\section{Mathematical models and solution approaches}

\subsection{Scenario 1}

As mentioned above, Sajadieh et al. (2008) have developed models for Scenarios 1 and 3. For convenience, we just review those models here.

\subsubsection{Buyer's cost formulation}

The buyer's expected long-run average cost is

$$
B C_{S 1}(r, Q)=\frac{A_{B} D}{Q}+h_{B}\left(r+\frac{Q}{2}-\frac{D}{\lambda}\right)+\frac{\left(h_{B}+\pi\right) D^{2}}{\lambda^{2} Q}\left(e^{\frac{-r \lambda}{D}}-e^{\frac{-(r+Q) \lambda}{D}}\right) .
$$

\subsubsection{Vendor's cost formulation}

The vendor's expected long-run average cost is

$$
V C_{S 1}(n)=\frac{A_{V} D}{n Q}+h_{V} \frac{Q}{2}\left((n-1)\left(1-\frac{D}{P}\right)+\frac{D}{P}\right) .
$$

In Scenario 1, each of the supply chain members optimizes its own policy. Thus the total expected cost in this scenario equals the sum of the buyer's and the vendor's costs, i.e., $T C_{S 1}(r, Q, n)=\min _{r, Q} B C_{S 1}(r, Q)+\min _{n} V C_{S 1}(n)$.

\subsection{Scenario 2}

In this scenario, two vendors are assumed to be identical in terms of their cost, production, and lead-time parameters. The order quantity is then split equally between the 
two vendors, and the split orders are placed simultaneously when the inventory position at the buyer's inventory reaches the reorder level.

\subsubsection{Buyer's cost formulation}

The expected long-run average cost function for the buyer under this dual-sourcing strategy has been developed by Ramasesh et. al (1991) as

$$
\begin{aligned}
B C_{S 2}(r, Q)= & \frac{(1+\alpha) A_{B} D}{Q}+h_{B}\left(r+\frac{Q}{2}-\frac{D}{\lambda}\right)+\frac{\left(h_{B}+\pi\right) D^{2}}{4 \lambda^{2} Q}\left(e^{\frac{-2 r \lambda}{D}}-2 e^{\frac{-(2 r+Q) \lambda}{D}}+e^{\frac{-2(r+Q) \lambda}{D}}\right) \\
& +\frac{2\left(h_{B}+\pi\right) D^{2}}{\lambda^{2} Q}\left(e^{\frac{-(2 r+Q) \lambda}{2 D}}-e^{\frac{-(r+Q) \lambda}{D}}\right),
\end{aligned}
$$

where $0 \leq \alpha \leq 1$ is a factor representing the percentage increase in the ordering cost of the buyer when moving from the sole to the dual sourcing strategy.

$B C_{S 2}(r, Q)$ is strictly and jointly convex in the reorder point and the order quantity (see Appendix A for the proof). Moreover, for a given value of $Q$ we can find a closed form solution for $r$ as

$$
r=-\frac{Q}{2}+\frac{D}{\lambda} \ln \left\{D\left(h_{B}+\pi\right)+\sqrt{D\left(h_{B}+\pi\right)\left(2 D\left(h_{B}+\pi\right)+h_{B} \lambda Q\left(1+e^{\frac{\lambda Q}{2 D}}\right)^{2} / 2\right)}\right\}+\frac{D}{\lambda} \ln \left(\frac{1-e^{\frac{-\lambda Q}{2 D}}}{h_{B} \lambda Q}\right) .
$$

Substituting expression (4) into the cost function (3), we obtain $B C_{S 2}(Q)$. As the cost function is convex, we can employ a one-dimensional search algorithm to compute the optimal value of $Q$.

\subsubsection{Vendor's cost formulation}

When the buyer's order quantity $Q$ is adopted, orders of size $Q / 2$ are received by each vendor at known intervals of length $T=Q / D$. From each vendor's point of view $D^{\prime}=D / 2$, $Q^{\prime}=Q / 2$ and $T=Q^{\prime} / D^{\prime}=Q / D$. Thus, in analogy with (2) the vendor's average inventory can be obtained as 


$$
A I_{V}=\frac{Q}{4}\left((n-1)\left(1-\frac{D}{2 P}\right)+\frac{D}{2 P}\right)
$$

Hence, the expected long-run average cost for each vendor is

$$
V C_{S 2}(n)=\frac{A_{V} D}{n Q}+h_{V} \frac{Q}{4}\left((n-1)\left(1-\frac{D}{2 P}\right)+\frac{D}{2 P}\right) .
$$

It is straightforward to show that $V C_{S_{2}}(n)$ is convex in $n$. (The same is evidently true for $V C_{S 1}(n)$ in Eq. (2).) Therefore, we obtain the following optimality conditions for the optimal number of shipments $n^{*}$

$$
n^{*}\left(n^{*}-1\right) \leq \frac{4 D A_{V}}{h_{V} Q^{2}(1-D / 2 P)} \leq n^{*}\left(n^{*}+1\right) .
$$

In Scenario 2, the buyer is free to choose the ordering policy $(r, Q)$, and the (identical) vendors are free to choose the number of shipments, $n$, per manufacturing batch. The total expected cost of Scenario 2, $T C_{S 2}(r, Q, n)$, then equals the sum of the buyer's and the two vendors' costs, i.e., $T C_{S 2}(r, Q, n)=\min _{r, Q} B C_{S 2}(r, Q)+2 \cdot \min _{n} V C_{S 2}(n)$.

\subsection{Scenario 3}

In this scenario, there is still one buyer and only one vendor. However, the two parties now cooperate and agree to follow the integrated jointly optimal replenishment policy. The expected long-run average cost of the buyer and the vendor, $T C_{S 3}(r, Q, n)$, is given by the sum of the costs in (1) and (2). Hence,

$$
\begin{aligned}
T C_{S 3}(r, Q, n)= & \frac{\left(n A_{B}+A_{V}\right) D}{n Q}+h_{B}\left(r+\frac{Q}{2}-\frac{D}{\lambda}\right)+\frac{D^{2}\left(h_{B}+\pi\right)}{\lambda^{2} Q}\left(e^{\frac{-r \lambda}{D}}-e^{\frac{-(r+Q) \lambda}{D}}\right) \\
& +h_{V} \frac{Q}{2}\left((n-1)\left(1-\frac{D}{P}\right)+\frac{D}{P}\right) .
\end{aligned}
$$

Sajadieh et al. (2008) have specified an algorithm for finding a solution $(r, Q, n)$ that minimizes the total cost in (7). 


\subsection{Scenario 4}

Now, suppose that all parties in Scenario 4, i.e., the two vendors and the buyer, cooperate and agree to follow the integrated and jointly optimal policy. The total expected long-run average cost for the buyer and the vendors, $T C_{S 4}(r, Q, n)$, is then given by

$$
\begin{aligned}
T C_{S 4}(r, Q, n)= & \frac{\left[n(1+\alpha) A_{B}+2 A_{V}\right] D}{n Q}+h_{B}\left(r+\frac{Q}{2}-\frac{D}{\lambda}\right)+\frac{D^{2}\left(h_{B}+\pi\right)}{4 \lambda^{2} Q}\left(e^{\frac{-2 r \lambda}{D}}-2 e^{\frac{-(2 r+Q) \lambda}{D}}+e^{\frac{-2(r+Q) \lambda}{D}}\right) \\
& +\frac{2 D^{2}\left(h_{B}+\pi\right)}{\lambda^{2} Q}\left(e^{\frac{-(2 r+Q) \lambda}{2 D}}-e^{\frac{-(r+Q) \lambda}{D}}\right)+h_{V} \frac{Q}{2}\left((n-1)\left(1-\frac{D}{2 P}\right)+\frac{D}{2 P}\right) .
\end{aligned}
$$

$T C_{S 4}(r, Q, n)$ is convex in $r$ and $Q$ for a given value of $n$. Moreover, it is convex in $n$ for given values of $r$ and $Q$. Thus, the optimality conditions (6) for $Q^{*}$ and $n^{*}$ are also valid in this scenario.

Additionally, it can be proven that $T C_{S 4}(r, Q, n)$ is convex in $r$ for given values of $Q$ and $n$. Taking the partial derivative of $T C_{S 4}(r, Q, n)$ with respect to $r$, and equating it to zero, we again obtain Eq. (4). Substituting into $T C_{S 4}(r, Q, n)$ and simplifying, we obtain $T C_{S 4}(Q, n)$. Hence, for a given $n$, the value of $Q$ that minimizes $T C_{S 4}(Q)$ can be obtained by a onedimensional search algorithm. Moreover, based on the following two lemmas, we can design a solution algorithm. In order to establish the algorithm, we first need to state and prove the two lemmas.

\section{Lemma 1}

Consider $Q^{*}$, the order quantity that minimizes $T C_{S 4}(Q, n)$ for a given value of $n$. If the number of shipments $n$ increases to $n+m$, where $m \in\{1,2,3, \ldots\}$, then the optimal order quantity will decrease to $Q^{*} / \beta k$, where $k=(n+m) / n$ and $n /(n+m)<\beta<1$. This implies that $Q$ and $n$ vary inversely and also that the decrease rate of the order quantity is lower than the increase rate of the number of shipments. 


\section{Lemma 2}

Consider Eq. (8) when solved for values of $n$ which are increased by one unit at a time. The increase of $n$ can be stopped when we arrive at a value for which the left-hand side of the optimality condition (6), $n(n-1)$, is greater than the threshold $\rho=4 D A_{v} / h_{V} Q^{2}(1-D / 2 P)$. The optimal number of shipments $n^{*}$ is less than this $n$, i.e., $n^{*}<n$.

The proofs of Lemmas 1 and 2 are provided in Appendix B and C, respectively.

\subsubsection{Solution algorithm}

Step 1. Initialize by setting $n=1$. Set $T C_{S 4}^{o p t}$ to a sufficiently large number.

Step 2. Find the value of $Q$ that minimizes $T C_{S 4}(Q)$ for the given value of $n$.

Step 3. Compute the resulting value of $r$ using Eq. (4).

Step 4. If $n(n-1)>4 D A_{v} / h_{V} Q^{2}(1-D / 2 P)$ then go to step 8 .

Step 5. If the conditions (6) are satisfied for $Q$ and $n$, then go to the next step. Otherwise, go to Step 7.

Step 6. If $T C_{S 4}(r, Q, n)<T C_{S 4}^{o p t}$, then set $T C_{S 4}^{o p t}=T C_{S 4}(r, Q, n), n_{o p t}=n, r_{o p t}=r$ and $Q_{o p t}=Q$.

Step 7. Increment $n$ by 1 and go to Step 2 .

Step 8. Stop, because the current solution is globally optimal.

\section{Numerical experiments and sensitivity analyses}

From numerical analyses using the models specified in Section 3 it is possible to find the best performing strategy for different situations. Intuitively, one might expect that the first scenario would be the worst for most cases, and that the forth scenario would be the best. The numerical results do not support this conclusion, but show that the worst case is usually the second scenario, and that the third scenario is usually the best. In fact, dual sourcing is not 
generally recommended when total system costs are considered. The reason will be discussed below.

Using the models in Section 3, we investigate the effects of lead-time variability as well as the buyer's backorder-to-holding cost, vendor-to-buyer ordering cost, and vendor-to-buyer holding cost ratios. The effects are evaluated by the impact on total costs in the four different scenarios, as well as the impact on the magnitude of the decision variables. To gain insight into these effects, different sets of parameter values are considered.

Referring to examples used in the literature, we first consider a base-case example with the following data: $D=1000 /$ year, $P=5000 /$ year, $A_{V}=\$ 400 /$ setup, $A_{B}=\$ 50 /$ order, $h_{V}=\$ 4 /$ unit/year, $h_{B}=\$ 7 /$ unit/year, $\pi=\$ 100 /$ unit/year, $1 / \lambda=30$ days, and $\alpha=0.15$. Variations of the base-case data are shown in Table 1. The ranges of parameters are defined so that the models remain valid, i.e., the probability of order crossover remains low. For the parameters used here, this probability is about $2.4 \%$ on average. The crossover possibility exists in all four scenarios and the same type of error is incurred when obtaining the total costs, although the order rate is somewhat higher in the dual sourcing scenarios. Hence, the approximation effect of crossovers will be dampened when comparing the scenarios with each other.

\section{Table 1}

\subsection{Effect of lead-time variation}

In order to analyze the effect of lead-time variation, we use eight levels for the lead-time parameter, i.e., $1 / \lambda \in\{5,10,15, \ldots, 40\}$. As the lead time is assumed to be exponentially distributed, the mean and standard deviation are equal. However, as demand is constant, a change in the mean lead time only, would just have a corresponding effect on the reorder point level. Thus, it can be concluded that variations in the lead-time parameter in Scenarios 1-4 lead to variations in the total expected costs caused by the changes in lead-time 
variability.

Figure 2 shows the total system costs of the four scenarios for different values of the leadtime parameter. The following conclusions can be discerned from Figure 2. First, the total cost in Scenario 1 closely follows the total cost in Scenario 3 for the whole range of the leadtime parameter. Obviously, Scenario 1 is dominated by Scenario 3. The same holds for Scenarios 2 and 4, where Scenario 4 dominates Scenario 2. As the lead-time variability increases, the savings percentage when comparing to the worst scenario (Scenario 2) decreases significantly for Scenarios 1 and 3, and changes only slightly for Scenario 4.

The cost increase rates for Scenarios 1 and 3 are higher than the increase rate for Scenario 4. When $1 / \lambda>35$, this makes the total cost in Scenario 4 smaller than that for the other scenarios. The reason is that as lead-time variability increases, the savings in backorder costs obtained by dual sourcing become more valuable. Thus, an indifference point $(I P)$ can be identified, where Scenarios 3 and 4 generate the same savings.

\section{Figure 2}

As mentioned above, the cost performance of Scenario 2 is the worst for most of the parameter ranges. This result contradicts results obtained in the order splitting literature. Therein, moving from sole to dual or multiple sourcing is found to be beneficial for a wide range of parameters (see, e.g. Ramasesh et. al, 1991). The reason is that in the papers published in the order splitting literature, the comparison between sole and dual sourcing is usually based on the buyer's costs only. Because of the effective lead-time decrease in dual sourcing compared to single sourcing, average inventory holding and backorder costs for the buyer decrease. Therefore, adding one more supplier will often be beneficial, when considering the buyer's costs only. However, in dual sourcing, three stocking points exist. The benefit of shifting to dual sourcing obtained by a decrease in the buyer's costs will be 
offset by increases in the vendors' costs. Hence, dual sourcing will be less attractive when considering the total system costs rather than the buyer's costs only.

Now we will look into whether it is more beneficial to have coordination between supply chain levels under a single sourcing or under a dual sourcing strategy. Moreover, we wish to establish whether the relative benefits are affected by the lead-time uncertainty.

In Figure 2, shifting from the non-cooperative to the cooperative supply chain is analyzed for the two different sourcing strategies. It can be seen that although cooperation (shifting from Scenario 1 to Scenario 3 or from Scenario 2 to Scenario 4) is always beneficial, it is relatively more effective when there are two suppliers. For the range of parameters considered, the average savings percentage obtained under the dual sourcing strategy is about 1.6 times bigger than under sole sourcing. In the dual sourcing strategy there are three stocking points. Therefore, coordination in this case will result in more savings on inventory costs.

Another conclusion that can be drawn from Figure 2 is that the benefits of coordination under both the sole and the dual sourcing strategies generally increase as the lead-time variability increases. In other words, coordination becomes more valuable as the environment becomes more unpredictable, i.e., as $1 / \lambda$ increases. (However, in the latter comparison, at $1 / \lambda=25$ there is an exception to the conclusion. This jump is related to a discrete change in the number of shipments that occurs at $1 / \lambda=25$.)

\subsection{Effect of setup-to-ordering costs ratio}

In Table 2, the optimal values of the decision variables in the four scenarios are compared to each other for different setup-to-ordering cost ratios $\mu=A_{V} / A_{B}$. As shown, Scenarios 1 and 3 have much higher reorder points than Scenarios 2 and 4. The reason is that in Scenarios 1 and 3 there is only one supplier, and to limit the backorder costs at the buyer, the system increases 
the safety stock and therefore the reorder point. In Scenarios 2 and 4, the effective lead time is decreased by pooling, and backorder costs can be partly controlled by order splitting.

Moreover, the total order quantity is smaller in Scenarios 1 and 3 than in Scenarios 2 and 4, respectively, because in Scenarios 2 and 4 the order quantity is split between two suppliers. This result is analogous to that obtained in the order splitting literature (see, e.g. Ramasesh, 1993).

Table 2 also shows that as the proportion of setup-to-ordering cost increases the order quantities in Scenarios 1 and 2 remain unchanged, while they generally increase in Scenarios 3 and 4. In Scenarios 1 and 2 there is no coordination between buyer and vendor(s), so the buyer does not take into account the increase in the manufacturing setup costs. Thus, the order/shipment quantities are unchanged. In Scenarios 3 and 4, the order quantity of the buyer is sensitive to the vendors' parameters. The production batch, $n Q$, always increases in the setup costs, and the order quantity $Q$ exhibits a decrease when moving from one value of $n$ to the next.

\section{Table 2}

The number of shipments is non-decreasing in $\mu$ for all scenarios. As the vendor's setup cost increases, more units will generally be produced in each setup. The number of shipments in Scenario 1 is equal to or larger than that in the other scenarios and the order quantity is lower. Moreover, the order quantity in Scenario 1 does not change by $\mu$. Hence, the number of shipments absorbs all of the increase related to a production batch increase.

The last two columns in Table 2 show the best sourcing strategy when considering the total system costs and when only the buyer's costs are considered. With a few exceptions, Scenario 3 is the preferred strategy when the total system costs are optimized. However, dual sourcing is always the best choice if the buyer makes decisions based only on his/her own 
costs. The latter case is the one often referred to in the order splitting literature.

A company that is presently using only one supplier may be interested in knowing whether it is better to coordinate with the existing supplier or employ another one at arm's length. In Figure 3, the cost savings percentages obtained from changing to the cooperative or to the dual sourcing strategy are illustrated by solid and dashed lines, respectively. Although cooperation with the supplier is always beneficial, the cost benefit is only about $1.2 \%$ on average. However, considering total system cost, it can be seen that dual sourcing is not an attractive option for a wide range of $\mu$ values. As the setup costs at the vendors increase, employing two vendors becomes more unfavorable in terms of total system costs. Hence, dual sourcing would be preferred only in situations in which lead time is quite variable and/or when costs at the vendor level are considerably lower than those that at the buyer's level. Seemingly, these results are in line with the trend towards consolidation of the supplier base inspired by the Just-In-Time (JIT) and Lean concepts.

Figure 3

It should also be noted that $\alpha$ has been assumed to be a fairly low $15 \%$ in the numerical examples. For higher values of $\alpha$, the ordering costs of Scenarios 2 and 4 would of course increase. These scenarios would then become even further dominated by Scenarios 1 and 3 .

\subsection{Effect of buyer's backorder-to-inventory cost ratio}

We now analyze the effect of the buyer's inventory cost as well as the backorder-to-inventory cost ratio on the scenario rankings. The importance of this analysis stems from the fact that different supply chains operate in different environments and deliver different types of products to the market. In some markets, competition is high and delivery delays result in very high costs. On the other hand, some products are more valuable, and therefore their holding cost is higher. Hence, we want to investigate whether one should select different 
sourcing strategies for products with higher inventory holding costs and/or higher shortage costs.

The answers to the above questions are summarized in Table 3. From Table 3 we note that as the inventory holding cost at the buyer $\left(h_{B}\right)$ increases, the best strategy eventually changes from Scenario 3 to Scenario 4. This trend is also evident when the ratio of backorder-to-inventory cost $\left(\omega=\pi / h_{B}\right)$ increases (for non-decreasing $h_{B}$ ). The reason is that as the inventory and shortage costs at the buyer increase, the scenarios with higher savings at the buyer's level become more important. Therefore, Scenario 4 becomes the best option. Increasing inventory holding and shortage costs even makes Scenario 2, which is usually dominated by other scenarios, the second best option. In other words, although coordination is always beneficial, if we are operating in a market with high shortage costs, adding one more supplier might be more valuable than coordination alone. Again, the dual sourcing strategy is always the best option if we limit the analysis to the buyer's costs only.

\section{Table 3}

\subsection{Effect of vendor's inventory-to-buyer's inventory holding cost ratio}

One of the standard assumptions in JELS literature is that the inventory holding cost at the buyer is higher than that at the vendor, i.e., $h_{B}>h_{V}$. This reflects the added product value as the product moves downstream in the distribution chain, and the associated holding cost is increased correspondingly. To see the impact of this assumption, the effect on the scenario costs of the inventory holding cost ratio parameter $\psi=h_{V} / h_{B}$ is analyzed next. As indicated in Table 1, eleven levels of $\psi$ are considered.

Figure 4(a) shows the difference between percentage savings for a shift to the dualsourcing strategy from the cooperative and non-cooperative strategies, respectively. The percentage cost savings difference is specified as 
$P S=\left[\left(T C_{S 3}-T C_{S 4}\right) / T C_{S 3}-\left(T C_{S 1}-T C_{S 2}\right) / T C_{S 1}\right] \times 100$. There is no simple relationship between $P S$ and $\psi$ when $\psi<0.6$. However, shifting to dual sourcing is $1.6 \%$ better on average with the cooperative strategy than with the non-cooperative strategy. As $\psi$ becomes small, shifting to dual souring with either the cooperative or the non-cooperative strategy yields almost the same cost savings. The reason is that if the inventory holding cost at the vendor is substantially decreased, it will be beneficial in all four scenarios to increase the production batch size considerably. Thus, the total cost differences between the scenarios become almost negligible.

However, for $\psi \geq 0.6$ an increasing trend can be identified for the $P S$. Because the average inventory at the vendor level is higher in non-cooperative strategies compared to cooperative strategies, establishing coordination becomes more valuable for higher values of $\psi$. The (negative) relative cost difference between the sole and dual sourcing strategies also increases (in absolute terms) with $\psi$ under a non-cooperative strategy. This difference is not significant under a cooperative strategy. Consequently, the difference between the percentage savings increases when shifting to the dual sourcing strategy from a cooperative strategy compared to from a non-cooperative strategy.

Similar effects can be observed in Figure 4(b), which shows the effects of a shift to the cooperative strategy from the sole and dual sourcing strategies, respectively.

\section{Figure 4}

\section{Sourcing strategies for non-identical suppliers}

One of the assumptions in Scenarios 2 and 4 is that the two vendors are identical in terms of their lead-time and cost parameters. In this section, we relax this assumption and investigate scenarios in which the vendors may have different lead-time means. We want to verify that the results obtained in the previous section are also valid for non-identical lead 
times. However, the cost parameters for both vendors are assumed to be the same, as before. Scenarios 2 and 4 under identical vendors are denoted as Scenarios 5 and 6, respectively, for non-identical vendors.

\subsection{Scenario 5}

In this scenario the two vendors are assumed to be non-identical in terms of their leadtime parameter, i.e., $L_{i} \sim \exp \left(\lambda_{i}\right), i=1,2$ for the $i$ th vendor. The order quantity is not necessarily split equally between the two vendors. In our two-supplier model, the order quantity is split between the vendors in the proportions $k_{1}$ and $k_{2}$, where $k_{1}+k_{2}=1$. The split orders are assumed to be placed simultaneously when the inventory position at the buyer reaches the reorder level, $r$.

\subsubsection{Buyer's cost formulation}

The expected long-run average cost function for the buyer under the dual-sourcing strategy with non-identical vendors is expressed by Ramasesh et. al (1993) as

$$
\begin{aligned}
B C_{S 5}(r, Q)=\frac{(1+\alpha) A_{B} D}{Q}+h_{B}\left(r+\frac{Q}{2}-D \sum_{i=1}^{2} \frac{k_{i}}{\lambda_{i}}\right)-\frac{D^{2}}{Q\left(\lambda_{1}+\lambda_{2}\right)^{2}} \sum_{\substack{i, j \in\{1,2\} \\
i \neq j}}\left(\frac{h_{B} \lambda_{i}}{\lambda_{j}}+\pi\right) e^{\frac{-\left(\lambda_{1}+\lambda_{2}\right)\left(r+k_{i} Q\right)}{D}} \\
+\frac{\left(h_{B}+\pi\right) D^{2}}{Q}\left[\frac{e^{-\frac{\left(\lambda_{1}+\lambda_{2}\right) r}{D}}\left(1+e^{-\frac{\left(\lambda_{1}+\lambda_{2}\right) Q}{D}}\right)}{\left(\lambda_{1}+\lambda_{2}\right)^{2}}+\sum_{i=1}^{2} \frac{e^{\frac{-\lambda_{i}(r+Q)}{D}\left(e^{\frac{\lambda_{i} k_{i} Q}{D}}-1\right)}}{\lambda_{i}^{2}}\right]-\frac{\pi D}{\lambda_{1} \lambda_{2}}\left(k_{1}-k_{2}\right)\left(\lambda_{1}-\lambda_{2}\right) e^{-\frac{\left(\lambda_{1}+\lambda_{2}\right)(r+Q)}{D}} .
\end{aligned}
$$

We assume that the proportion of the order to each vendor is pre-specified based on an overall purchase allocation strategy of the buyer. Therefore, $k_{1}$ (and thus $k_{2}$ ) is considered as a given value in this analysis. Nevertheless, the Hessian matrix turns out to be very complex even in this case, and convexity is therefore difficult to prove analytically. Hence, we resort to an extensive grid search to analyze the convexity of the expected cost function. The results show that $B C_{S 5}(r, Q)$ appears to be jointly convex in $r$ and $Q$, when the order quantity $Q$ is large enough. For small values of the order quantity, the model is not valid because the 
resulting order crossover probability becomes too high.

\subsubsection{Vendor's cost formulation}

When the buyer's order quantity $Q$ is determined, orders of size $k_{1} Q$ and $k_{2} Q$ are received by the first and second vendor, respectively. The time interval between orders is $T=Q / D$. From the $i$ th vendor's point of view $D_{i}^{\prime}=k_{i} D, Q_{i}^{\prime}=k_{i} Q$ and $T_{i}=Q_{i}^{\prime} / D_{i}^{\prime}=Q / D$. Thus, in analogy with (2) the total average cost for the $i$ th vendor is obtained as

$$
V C_{S 5}\left(n_{i}\right)=\frac{D A_{V}}{n_{i} Q}+h_{V} \frac{k_{i} Q}{2}\left(\left(n_{i}-1\right)\left(1-\frac{k_{i} D}{P}\right)+\frac{k_{i} D}{P}\right) .
$$

$V C_{S 5}\left(n_{i}\right)$ is convex in $n_{i}$. Therefore, we obtain the following optimality conditions for the optimal number of shipments $n_{i}^{*}$

$$
n_{i}^{*}\left(n_{i}^{*}-1\right) \leq \frac{2 D A_{V}}{h_{V} k_{i} Q^{2}\left(1-k_{i} D / P\right)} \leq n_{i}^{*}\left(n_{i}^{*}+1\right) .
$$

As in Scenario 2, the buyer chooses the ordering policy, $(r, Q)$, and each of the vendors chooses their preferred number of shipments, $n_{i}$. The total expected cost of Scenario 5 , $T C_{S 5}\left(r, Q, n_{1}, n_{2}\right)$, is then equal to the sum of the buyer's and the two vendors' expected costs, i.e., $T C_{S 5}\left(r, Q, n_{1}, n_{2}\right)=\min _{r, Q} B C_{S 5}(r, Q)+\min _{n_{1}} V C_{S 5}\left(n_{1}\right)+\min _{n_{2}} V C_{S 5}\left(n_{2}\right)$.

\subsection{Scenario 6}

If the two vendors and the buyer agree to follow the jointly optimal policy, then the total expected long-run average cost, $T C_{S 6}\left(r, Q, n_{1}, n_{2}\right)$, is given by

$$
\begin{aligned}
& T C_{S 6}\left(r, Q, n_{1}, n_{2}\right)=\frac{(1+\alpha) D A_{B}}{Q}+h_{B}\left(r+\frac{Q}{2}-D \sum_{i=1}^{2} \frac{k_{i}}{\lambda_{i}}\right)-\frac{D^{2}}{Q\left(\lambda_{1}+\lambda_{2}\right)^{2}} \sum_{\substack{i, j \in\{1,2\} \\
i \neq j}}\left(\frac{h_{B} \lambda_{i}}{\lambda_{j}}+\pi\right) e^{\frac{-\left(\lambda_{1}+\lambda_{2}\right)\left(r+k_{i} Q\right)}{D}} \\
&+ \frac{\left(h_{B}+\pi\right) D^{2}}{Q}\left[\frac{e^{-\frac{\left(\lambda_{1}+\lambda_{2}\right) r}{D}}\left(1+e^{-\frac{\left(\lambda_{1}+\lambda_{2}\right) Q}{D}}\right)}{\left(\lambda_{1}+\lambda_{2}\right)^{2}}+\sum_{i=1}^{2} \frac{e^{\frac{-\lambda_{i}(r+Q)}{D}}\left(e^{\frac{\lambda_{i} k_{i} Q}{D}}-1\right)}{\lambda_{i}^{2}}\right] \\
&-\frac{\pi D}{\lambda_{1} \lambda_{2}}\left(k_{1}-k_{2}\right)\left(\lambda_{1}-\lambda_{2}\right) e^{-\frac{\left(\lambda_{1}+\lambda_{2}\right)(r+Q)}{D}}+\sum_{i=1}^{2}\left(\frac{D A_{V}}{n_{i} Q}+h_{V} \frac{k_{i} Q}{2}\left(\left(n_{i}-1\right)\left(1-\frac{k_{i} D}{P}\right)+\frac{k_{i} D}{P}\right)\right) .
\end{aligned}
$$


Numerical search is used to find the values of $r$ and $Q$ that minimize $T C_{S 6}\left(r, Q, n_{1}, n_{2}\right)$ for given values of $n_{1}$ and $n_{2}$. An extensive numerical search is then also conducted to find the optimal number of shipments for $n_{1}$ and $n_{2}$.

\subsection{Sensitivity analyses}

In order to analyze the effects of non-identical lead times, six levels of the lead-time parameter have been specified for the first vendor, i.e. $1 / \lambda_{1} \in\{5,10,15, \ldots, 30\}$. Figure 5 shows the average percentage cost savings $P S_{i}$ obtained by using Scenarios 1, 3, and 6 compared to using Scenario 5, where $P S_{i}=\left(T C_{S 5}-T C_{S i}\right) / T C_{S 5} \times 100, \quad i=1,3,6$. The results shown in Figure 5 are the averages of six cases in which $k_{1} \in\{0.5,0.6, \ldots, 1\}$, and $1 / \lambda_{2}=30$.

\section{Figure 5}

One of the results obtained in Section 4 was that dual sourcing is often not the preferred strategy when total system costs are considered. Comparing Figure 5 with Figure 2, we see that the results obtained here are the same as those obtained in the case of identical vendors. As lead-time variability increases, the savings percentage obtained when comparing with Scenario 5 decreases for both Scenarios 1 and 3. However, it remains relatively stable for Scenario 6 . The average savings percentage obtained by shifting to Scenarios 1 or 3 is about $14 \%$, whereas it is only about $1.5 \%$ when shifting to Scenario 6 . Therefore, in this case shifting to sole sourcing is more critical than establishing a close cooperation.

Figure 5 also shows that when sourcing from non-identical vendors, shifting to the solesourcing strategy becomes an even more attractive option than in the case of identical vendors. That is caused by the fact that in sole sourcing (Scenarios 1 and 3), the buyer chooses the best vendor. Evidently, the case of two non-identical vendors (Scenario 5) is always dominated by two identical vendors when both of them are assumed to have the same 
characteristics as the best vendor (Scenario 2). Moreover, it was shown before that Scenario 2 is often not the preferred option. Thus, Scenario 5 is not a preferred option either, and this indicates that the conclusions drawn earlier in this paper are valid for the case of nonidentical vendors too.

Above, the order split proportion was based on the buyer's presumed purchasing strategy. In Figure 6, we investigate the impact of this decision on the scenario costs. Obviously, the costs in Scenarios 1 and 3 are independent of the split proportion. Because Scenario 6 always dominates Scenario 5, we concentrate on the cost effect of $k_{1}$ on Scenario 6 . Figure 6 illustrates the percentage cost savings $P S$ obtained by shifting from Scenario 6 to Scenario 3, where $P S=\left(T C_{S 6}-T C_{S 3}\right) / T C_{S 6} \times 100$. The mean lead time of the second vendor is kept constant at $1 / \lambda_{2}=30$.

Figure 6 shows that changes in $k_{1}$ have a limited effect on $P S$ for some cases with a small difference between the lead times of the two vendors. Consider the case in which the lead time of the first vendor is $1 / \lambda_{1}=25$. When increasing $k_{1}$ from $50 \%$ to $90 \%$, PS increases only from about $8.0 \%$ to about $9.9 \%$. In other cases, such as when the difference in the mean lead time is larger, the effect on $P S$ from changes in $k_{1}$ is more pronounced.

\section{Figure 6}

Figure 6 indicates some interesting results with respect to the order splitting decision. The benefit of shifting to sole sourcing (Scenario 3) decreases in the split proportion when $1 / \lambda_{1}=5$ and $1 / \lambda_{1}=15$. This also suggests that the preferred split proportion is the highest possible in Scenario 6. However, when $1 / \lambda_{1}=25$ the optimal split ratio in Scenario 6 seems to be around $60 \%$. When $1 / \lambda_{1}=30$, i.e., with identical vendors, the optimal split ratio becomes $50 \%$, as expected. 


\section{Conclusions and discussion}

Coordination is one of the main activities in supply chain management. The analyses of the different sourcing models in this paper provide insights for companies to find the best strategy with respect to vertical coordination and/or multiple sourcing. For this purpose, we have developed four different scenarios and specified analytical and numerical solution procedures to determine optimal replenishment policies. Moreover, we have conducted a comprehensive numerical study to investigate the sensitivity of each scenario to endogenous and exogenous changes. In this way we have shown how cost parameters and supply conditions affect the best choice of strategy. The main conclusions are summarized below.

First, we conclude that in many cases the recommendations found in the order-splitting literature do not seem to be valid, when total system costs are considered. For a wide range of parameter values, changing from sole to dual sourcing is not found to be beneficial for the supply chain dyad. This result also confirms one of the conjectures formulated by Thomas and Tyworth (2006) in their critique of the order splitting literature.

Second, although our study shows that there is no overall dominating scenario, Scenario 3 is often the best option. In other words, our models suggest that primarily a single supplier/vendor strategy should be sought, whereupon close coordination with the chosen vendor should be considered. This conclusion is also in line with the trend towards integration with suppliers inspired by the Just-In-Time (JIT) and Lean concepts. However, it is well-known that coordination is always beneficial in supply chain models of the type employed here. Thus, if a dual sourcing strategy has been chosen, it is also worthwhile to consider coordination with the vendors.

Third, the dual sourcing scenarios are suggested only for the situations in which a high proportion of the total system costs are related to the buyer's stage. For example, Scenarios 2 or 4 should be selected only when one or several of the following situations occur: The lead 
time is highly variable, delivery delays result in very high shortage costs, the vendors' setup costs are low, and holding costs are considerably higher for the buyer than for the vendor.

Fourth, with two non-identical vendors with different lead-time parameters, Scenario 3 is again the preferred option in most cases. In fact, the desirability of the sole sourcing strategy increases even further in the case of two non-identical vendors.

The cost comparisons that form the basis for the conclusions above do not include the costs for establishing or maintaining the cooperative mechanisms in Scenarios 3 and 4. Such costs would typically be costs for obtaining, monitoring, sharing, and using information, as well as additional costs for necessary profit sharing arrangements. It would be reasonable to expect such costs to be higher in Scenario 4 than in Scenario 3. Moreover, it should be noted that strategically Scenario 4 might not be a realistic alternative in many settings, because it would involve close collaboration with two possibly competing vendors. Such collaborative arrangements may not be easy to establish, although it could be a viable option if the different vendors are organizationally related, or if the buyer has strong channel power. However, Scenario 4 is also of interest in itself for benchmarking purposes, as in this paper.

Clearly, the models studied here could be extended towards more general situations, such as stochastic demands, stochastic prices, and other distributions for the lead times. Developing multi-supplier or multiple-tier models is also a possible future research direction.

\section{Acknowledgment}

The paper was written while the first author was a visiting scholar at CORAL - Centre for Operations Research Applications in Logistics at Aarhus School of Business, Aarhus University. The second author's work has been partly supported by grant no. 275-07-0094 from the Danish Social Science Research Council. 


\section{Appendix A}

We provide a proof that the Hessian matrix $G$ associated with $B C_{S 2}(r, Q)$ (see Eq. (3) in Section 3.2.1) is positive definite and thus that this function is strictly and jointly convex in $r$ and $Q$. Consider $G$ as the matrix

$$
G=\left[\begin{array}{ll}
g_{11} & g_{12} \\
g_{21} & g_{22}
\end{array}\right]
$$

where $g_{i j}(i, j=1,2)$ is the second partial derivative. We have

$$
g_{11}=\frac{\partial^{2}(T C)}{\partial r^{2}}=\frac{\left(h_{B}+\pi\right)}{Q}\left[e^{\frac{-2 r \lambda}{Q}}\left(1-2 e^{\frac{-Q \lambda}{D}}+e^{\frac{-2 Q \lambda}{D}}\right)+2 e^{\frac{-r \lambda}{Q}}\left(e^{\frac{-Q \lambda}{2 D}}-e^{\frac{-Q \lambda}{D}}\right)\right] .
$$

The Hessian determinants $\left(\left|G_{1}\right|\right.$ and $\left.|G|\right)$ can be obtained as follows:

$\left|G_{1}\right|=g_{11}$

The second expression in $g_{11}$ is positive, so we need to show that $1-2 e^{\frac{-Q \lambda}{D}}+e^{\frac{-2 Q \lambda}{D}} \geq 0$ to conclude that $\left|G_{1}\right|=g_{11}>0$. Let $x=e^{\frac{-Q \lambda}{D}}$. Then we have $1-2 e^{\frac{-Q \lambda}{D}}+e^{\frac{-2 Q \lambda}{D}}=1-2 x+x^{2}=(1-x)^{2}$. Thus, $\left|G_{1}\right|=g_{11}>0$. Finally,

$$
|G|=g_{11} g_{22}-g_{12} g_{21}=\frac{\left(h_{B}+\pi\right)^{2}}{4 Q^{4} \lambda^{2}} \gamma_{1}+\frac{2 A_{B}(1+\alpha) D\left(h_{B}+\pi\right)}{Q^{4}} \gamma_{2},
$$

where

$$
\begin{aligned}
\gamma_{1}= & 2\left(6 D^{2}+\lambda^{2} Q^{2}+2 D \lambda Q\right)\left(2 e^{\frac{-(3 r+2 Q) \lambda}{D}}+e^{\frac{-(6 r+Q) \lambda}{2 D}}\right)-2\left(2 D^{2}+\lambda^{2} Q^{2}\right) e^{\frac{-(4 r+3 Q) \lambda}{D}}-4\left(8 D^{2}+\lambda^{2} Q^{2}\right) e^{\frac{-(4 r+3 Q) \lambda}{2 D}} \\
& -4\left(3 D^{2}+2 \lambda^{2} Q^{2}+2 D \lambda Q\right) e^{\frac{-(3 r+Q) \lambda}{D}}+2\left(6 D^{2}+\lambda^{2} Q^{2}-2 D \lambda Q\right) e^{\frac{-(6 r+5 Q) \lambda}{2 D}}+2\left(3 D^{2}+2 \lambda^{2} Q^{2}\right) e^{\frac{-(4 r+2 Q) \lambda}{D}} \\
& -2\left(2 D^{2}+\lambda^{2} Q^{2}\right) e^{\frac{-(4 r+Q) \lambda}{D}}+D^{2}\left(e^{\frac{-4 r \lambda}{D}}-12 e^{\frac{-3(r+Q) \lambda}{D}}+16 e^{\frac{-(2 r+Q) \lambda}{D}}+16 e^{\frac{-2(r+Q) \lambda}{D}}-24 e^{\frac{-3(2 r+Q) \lambda}{2 D}}+e^{\frac{-4(r+Q) \lambda}{D}}\right),
\end{aligned}
$$

and $\gamma_{2}=e^{\frac{-2 r \lambda}{Q}}\left(1-2 e^{\frac{-Q \lambda}{D}}+e^{\frac{-2 Q \lambda}{D}}\right)+2 e^{\frac{-r \lambda}{Q}}\left(e^{\frac{-Q \lambda}{2 D}}-e^{\frac{-Q \lambda}{D}}\right)$.

It was shown above that $\gamma_{2}>0$, so if $\gamma_{1} \geq 0$, then we can conclude that $|G|>0$. Letting $x=e^{\frac{-Q \lambda}{D}}$ and $y=e^{\frac{-r \lambda}{D}}$, we can rewrite $\gamma_{1}$ as $\gamma_{1}=D^{2} y^{2} \theta$, where

$$
\begin{aligned}
\theta= & 12 y \sqrt{x}(1-\sqrt{x})(1-x)^{2}+y^{2}(1-x)^{4}+16 x(1-\sqrt{x})^{2}-4 y \sqrt{x}(1-\sqrt{x})^{2}(1-x) \ln x \\
& -2 y^{2} x(1-x)^{2} \ln ^{2} x-4 x \sqrt{x} \ln ^{2} x+2 y \sqrt{x}\left(1-4 \sqrt{x}+x^{2}+2 x^{3 / 2}\right) \ln ^{2} x .
\end{aligned}
$$


For $Q>0$ and $r \geq 0$, we have $0 \leq x \leq 1$ and $0 \leq y \leq 1$. The proof of non-negativity of $\theta$ is very complex. However, it can be shown graphically that $\theta \geq 0$ for the relevant ranges of $x$ and $y$. Figure 7 shows values of $\theta$ for $0 \leq x \leq 1$ and $0 \leq y \leq 1$. As seen in this figure, the value of $\theta$ is always positive for these ranges of $x$ and $y$. Thus, $|G|>0$. Hence, the Hessian matrix $G$ is positive definite and the function $B C_{S 2}(r, Q)$ is strictly and jointly convex in $r$ and $Q$.

\section{Figure 7}

\section{Appendix B. Proof of Lemma 1}

The total expected cost in Section 3.4 can be rewritten as $T C_{S 4}(Q, n)=T C_{S 4}^{1}+T C_{S 4}^{2}$, where $T C_{S 4}^{1}=2 A_{V} D / n Q+h_{V} n Q(1-D / 2 P) / 2$. Thus, $T C_{S 4}^{2}$ is independent of the number of shipments $n$. If the number of shipments increases to $n+m$, the effect on the optimal order quantity from $T C_{S 4}^{1}$ will at most be a decrease to $Q^{*} / k$, where $k=(n+m) / n$. Moreover, because $T C_{S 4}^{2}$ is independent of the number of shipments, the optimal order quantity is unaffected by this part of the total cost. Therefore, the new optimal order quantity for the summation of these two cost functions, $Q_{\text {new }}$, will be in the interval $Q^{*} / k<Q_{\text {new }}<Q^{*} / 1$. In other words, $Q_{\text {new }}=Q^{*} / \beta k$, where $1 / k<\beta<1$.

\section{Appendix C. Proof of Lemma 2}

We need to prove that the increase rate of the lower limit $L L$ of the optimality conditions (6) is higher than $\rho=4 A_{V} D / h_{V} Q^{2}(1-D / 2 P)$, as the number of shipments, $n$, increases. Then we can conclude that there will be a point (number of shipments) such as $n^{\prime}$ at which $\rho<L L$. If the increase rate of $L L$ is faster than $\rho$, the inequality $\rho<L L$ remains valid for all number of shipments larger than $n^{\prime}$, and the optimality conditions (6) will not be satisfied for any $n \geq n^{\prime}$. Hence, the optimal number of shipments must be less than $n^{\prime}$. 
Assume that the number of shipments is increased from $n$ to $n+1$. The increase rate of $L L$ can then be obtained as

$$
I R_{L L}=\frac{L L_{n+1}-L L_{n}}{L L_{n}}=\frac{(n+1) n-n(n-1)}{n(n-1)}=\frac{2}{n-1} .
$$

Based on Lemma 1, if the number of shipments increases from $n$ to $n+1$, the order quantity will decrease to $Q / \beta k$, where $1<\beta k<(n+1) / n(=k)$. Consequently, the increase rate of $\rho$ can be obtained as

$$
I R_{\rho}=\frac{\rho_{n+1}-\rho_{n}}{\rho_{n}}=\frac{\beta^{2} k^{2}\left[4 A_{V} D / h_{V} Q^{2}(1-D / 2 P)\right]-4 A_{V} D / h_{V} Q^{2}(1-D / 2 P)}{4 A_{V} D / h_{V} Q^{2}(1-D / 2 P)}=\beta^{2} k^{2}-1
$$

As noted above, $\beta k<(n+1) / n$. Thus,

$$
I R_{\rho}=\beta^{2} k^{2}-1<\frac{2 n+1}{n^{2}} .
$$

It can easily be shown that $(2 n+1) / n^{2}<2 /(n-1), \forall n \geq 1$, and thus $I R_{\rho}<I R_{L L}$. This means that the lower limit of the optimality conditions increases faster than $\rho$. As the number of shipments increases, we will eventually arrive at a point at which $\rho<L L$.

\section{References}

Banerjee, A. (1986). A joint economic lot-size model for purchaser and vendor, Decision Science, 17, 292-311.

Ben-Daya, M., Darwish, M., Ertogral, K. (2008). The joint economic lot sizing problem: review and extensions, European Journal of Operational Research, 185, 726-742.

Chiang C., Benton W.C. (1994). Sole sourcing versus dual sourcing under stochastic demands and lead times, Naval Research Logistics, 41, 609-24.

Goyal, S.K. (1976). An integrated inventory model for a single supplier-single customer problem, International Journal of Production Research, 15 (1), 107-111.

Goyal, S.K. (1988). A joint economic lot-size model for purchaser and vendor: A comment, Decision Science, 19, 236-241. 
Goyal, S.K. (1995). A one-vendor multi-buyer integrated inventory model: A comment, European Journal of Operational Research, 82, 209-210.

Hill R.M. (1996). Order splitting in continuous review $(Q, r)$ inventory models, European Journal of Operational Research, 95, 53-61.

Hill, R.M. (1997). The single-vendor, single-buyer integrated production-inventory model with a generalized policy, European Journal of Operational Research, 97, 493-499.

Hill, R.M. (1999). The optimal production and shipment policy for the single-vendor single-buyer integrated production-inventory model, International Journal of Production Research, 37, 24632475.

Lau H.S., Zhao L.G. (1993). Optimal ordering policies with two suppliers when lead times and demands are all stochastic, European Journal of Operational Research, 68(1), 120-33.

Minner, S. (2003). Multiple-supplier inventory models in supply chain management: a review, International Journal of Production Economics, 81-82, 265-79.

Muson, C.L., Rosenblatt, M.J. (2001). Coordinating a three-level supply chain with quantity discounts, IIE Transactions, 33, 371-384.

Huang, C.K. (2004). An optimal policy for a single-vendor, single-buyer integrated production inventory problem with process unreliability consideration, International Journal of Production Economics 91, 91-98.

Ramasesh R.V., Ord J.K., Hayya J.C., Pan A. (1991). Sole versus dual sourcing in stochastic leadtimes $(s, Q)$ inventory models, Management Science, 37(4), 428-43.

Ramasesh R.V., Ord J.K., Hayya J.C. (1993). Note: dual sourcing with non-identical suppliers, Naval Research Logistics, 40, 279-88.

Ryu S.W., Lee K.K. (2003). A stochastic inventory model of dual sourced supply chain with leadtime reduction, International Journal of Production Economics, 81-82, 513-24.

Sajadieh, M.S., Jokar, M.R.A., Modarres, M. (2008). Developing a coordinated vendor-buyer model in two-stage supply chains with stochastic lead times, Computers \& Operations Research, doi: 10.1016/j.cor.2008.10.001.

Sculli D., Wu S.Y. (1981). Stock control with two suppliers and normal lead times, Journal of the Operational Research Society, 32, 1003-09.

Sculli D., Shum Y.W. (1990). Analysis of a continuous review stock-control model with multiple suppliers, Journal of the Operational Research Society, 41(9), 873-77. 
Sedarage D., Fujiwara O., Luong H.T. (1999). Determining optimal order splitting and reorder levels for $n$-supplier inventory systems, European Journal of Operational Research, 116, 389-404.

Thomas D.J., Tyworth J.E. (2006). Pooling lead-time risk by order splitting: A critical review, Transportation Research Part E, 42, 245-57.

Toptal, A., Cetinkaya, S., Lee, C.Y. (2003). The buyer-vendor coordination problem: modeling inbound and outbound cargo capacity and costs, IIE Transactions, 35, 987-1002.

Yang, P.C., Wee, H.M. (2000). Economic ordering policy of deteriorated item for vendor and buyer: an integrated approach, Production Planning \& Control, 11(5), 474-480. 


\section{Figure Captions}

- Figure 1. Four sourcing scenarios obtained from combination of sourcing and coordination strategies

- Figure 2. Effect of lead-time variation on scenario rankings

- Figure 3. Effect of ordering and setup costs on scenario shifts

- Figure 4. Effect of $\psi$ on scenario shifts

- Figure 5. Effect of lead-time variation on percentage cost savings relative to Scenario 5

- Figure 6. Effect of order split proportion $k_{1}$ on percentage cost savings when shifting from Scenario 6 to Scenario 3

- Figure 7. Value of $\theta$ for the full ranges of parameters $x$ and $y$ 


\section{Table 1}

Table 1. Parameter ranges used in sensitivity analyses

\begin{tabular}{c|c}
\hline Parameters & Variation range \\
\hline$h_{B}$ & $\{4,7,10\}$ \\
$A_{B}$ & $\{50,75,100\}$ \\
$1 / \lambda$ & $\{5,10,15, \ldots, 40\}$ \\
$h_{V} / h_{B}$ & $\{0.0,0.1,0.2, \ldots, 1.0\}$ \\
$A_{V} / A_{B}$ & $\{1,4,8,12, \ldots, 28\}$ \\
$\pi / h_{B}$ & $\{4,8,12, \ldots, 28\}$
\end{tabular}

\section{Table 2}

Table 2. Effect of ordering and setup costs on decision variables and best choice of Scenarios 1-4

\begin{tabular}{|c|c|c|c|c|c|c|c|c|c|c|c|c|c|c|c|}
\hline \multicolumn{2}{|c|}{ Parameters } & \multicolumn{3}{|c|}{ Scenario 1} & \multicolumn{3}{|c|}{ Scenario 2} & \multicolumn{3}{|c|}{ Scenario 3} & \multicolumn{3}{|c|}{ Scenario 4} & \multirow{2}{*}{$\begin{array}{l}\text { System's } \\
\text { best } \\
\text { scenario }\end{array}$} & \multirow{2}{*}{$\begin{array}{c}\text { Buyer's } \\
\text { best } \\
\text { strategy }\end{array}$} \\
\hline$A_{B}$ & $\mu$ & $r$ & $Q$ & $n$ & $r$ & $Q$ & $n$ & $r$ & $Q$ & $n$ & $r$ & $Q$ & $n$ & & \\
\hline \multirow[t]{8}{*}{50} & 1 & 144.3 & 197.4 & 1 & 58.9 & 304.7 & 1 & 133.9 & 231.8 & 1 & 45.9 & 350.3 & 1 & 4 & Dual \\
\hline & 4 & 144.3 & 197.4 & 2 & 58.9 & 304.7 & 2 & 108.2 & 330.6 & 1 & 23.7 & 458.9 & 1 & 4 & Dual \\
\hline & 8 & 144.3 & 197.4 & 3 & 58.9 & 304.7 & 2 & 125.1 & 263.1 & 2 & 40.4 & 372.7 & 2 & 3 & Dual \\
\hline & 12 & 144.3 & 197.4 & 3 & 58.9 & 304.7 & 3 & 114.5 & 304.1 & 2 & 30.2 & 421.3 & 2 & 3 & Dual \\
\hline & 16 & 144.3 & 197.4 & 4 & 58.9 & 304.7 & 3 & 106.3 & 338.9 & 2 & 23.0 & 463.1 & 2 & 3 & Dual \\
\hline & 20 & 144.3 & 197.4 & 4 & 58.9 & 304.7 & 3 & 123.1 & 270.4 & 3 & 17.5 & 500.5 & 2 & 3 & Dual \\
\hline & 24 & 144.3 & 197.4 & 4 & 58.9 & 304.7 & 4 & 117.9 & 290.6 & 3 & 34.3 & 400.8 & 3 & 3 & Dual \\
\hline & 28 & 144.3 & 197.4 & 5 & 58.9 & 304.7 & 4 & 113.3 & 309.2 & 3 & 29.8 & 423.6 & 3 & 3 & Dual \\
\hline \multirow[t]{8}{*}{75} & 1 & 135.0 & 228.0 & 1 & 53.0 & 324.1 & 1 & 123.0 & 270.8 & 1 & 37.8 & 384.4 & 1 & 4 & Dual \\
\hline & 4 & 135.0 & 228.0 & 2 & 53.0 & 324.1 & 2 & 95.7 & 388.7 & 1 & 14.8 & 521.1 & 1 & 4 & Dual \\
\hline & 8 & 135.0 & 228.0 & 3 & 53.0 & 324.1 & 3 & 112.3 & 313.2 & 2 & 29.0 & 427.7 & 2 & 3 & Dual \\
\hline & 12 & 135.0 & 228.0 & 3 & 53.0 & 324.1 & 3 & 101.2 & 362.4 & 2 & 19.3 & 487.6 & 2 & 3 & Dual \\
\hline & 16 & 135.0 & 228.0 & 4 & 53.0 & 324.1 & 4 & 116.1 & 297.7 & 3 & 33.2 & 405.8 & 3 & 3 & Dual \\
\hline & 20 & 135.0 & 228.0 & 4 & 53.0 & 324.1 & 4 & 109.7 & 324.4 & 3 & 27.0 & 439.0 & 3 & 3 & Dual \\
\hline & 24 & 135.0 & 228.0 & 5 & 53.0 & 324.1 & 4 & 104.2 & 348.7 & 3 & 22.0 & 469.3 & 3 & 3 & Dual \\
\hline & 28 & 135.0 & 228.0 & 5 & 53.0 & 324.1 & 5 & 117.1 & 293.9 & 4 & 17.9 & 497.5 & 3 & 3 & Dual \\
\hline \multirow[t]{8}{*}{100} & 1 & 127.9 & 252.9 & 1 & 48.4 & 340.8 & 1 & 114.8 & 302.9 & 1 & 31.7 & 413.5 & 1 & 4 & Dual \\
\hline & 4 & 127.9 & 252.9 & 2 & 48.4 & 340.8 & 2 & 86.4 & 437.0 & 1 & 8.5 & 575.9 & 1 & 3 & Dual \\
\hline & 8 & 127.9 & 252.9 & 3 & 48.4 & 340.8 & 3 & 102.8 & 354.8 & 2 & 21.3 & 474.2 & 2 & 3 & Dual \\
\hline & 12 & 127.9 & 252.9 & 3 & 48.4 & 340.8 & 3 & 91.3 & 410.8 & 2 & 12.0 & 544.6 & 2 & 3 & Dual \\
\hline & 16 & 127.9 & 252.9 & 4 & 48.4 & 340.8 & 4 & 106.4 & 338.8 & 3 & 24.5 & 453.8 & 3 & 3 & Dual \\
\hline & 20 & 127.9 & 252.9 & 4 & 48.4 & 340.8 & 4 & 99.7 & 369.3 & 3 & 18.6 & 492.3 & 3 & 3 & Dual \\
\hline & 24 & 127.9 & 252.9 & 5 & 48.4 & 340.8 & 5 & 94.0 & 397.1 & 3 & 14.0 & 527.7 & 3 & 3 & Dual \\
\hline & 28 & 127.9 & 252.9 & 5 & 48.4 & 340.8 & 5 & 107.1 & 335.5 & 4 & 25.3 & 449.2 & 4 & 3 & Dual \\
\hline
\end{tabular}


Table 3

Table 3. Effect of buyer's holding and backorder costs on scenario

\begin{tabular}{|c|c|c|c|c|c|c|}
\hline \multicolumn{2}{|c|}{ Parameters } & \multicolumn{4}{|c|}{ Strategy ranking } & \multirow{2}{*}{$\begin{array}{c}\text { Buyer's } \\
\text { best } \\
\text { strategy }\end{array}$} \\
\hline$h_{B}$ & $\omega$ & First & Second & Third & Fourth & \\
\hline \multirow[t]{7}{*}{4} & 4 & 3 & 1 & 4 & 2 & Dual \\
\hline & 8 & 3 & 1 & 4 & 2 & Dual \\
\hline & 12 & 3 & 1 & 4 & 2 & Dual \\
\hline & 16 & 3 & 1 & 4 & 2 & Dual \\
\hline & 20 & 3 & 1 & 4 & 2 & Dual \\
\hline & 24 & 3 & 4 & 1 & 2 & Dual \\
\hline & 28 & 3 & 4 & 1 & 2 & Dual \\
\hline \multirow[t]{7}{*}{7} & 4 & 3 & 1 & 4 & 2 & Dual \\
\hline & 8 & 3 & 1 & 4 & 2 & Dual \\
\hline & 12 & 3 & 1 & 4 & 2 & Dual \\
\hline & 16 & 3 & 1 & 4 & 2 & Dual \\
\hline & 20 & 3 & 4 & 1 & 2 & Dual \\
\hline & 24 & 3 & 4 & 1 & 2 & Dual \\
\hline & 28 & 4 & 3 & 2 & 1 & Dual \\
\hline \multirow[t]{7}{*}{10} & 4 & 3 & 1 & 4 & 2 & Dual \\
\hline & 8 & 3 & 1 & 4 & 2 & Dual \\
\hline & 12 & 4 & 3 & 1 & 2 & Dual \\
\hline & 16 & 4 & 2 & 3 & 1 & Dual \\
\hline & 20 & 4 & 2 & 3 & 1 & Dual \\
\hline & 24 & 4 & 2 & 3 & 1 & Dual \\
\hline & 28 & 4 & 2 & 3 & 1 & Dual \\
\hline
\end{tabular}




\section{Figure 1}

\begin{tabular}{l|c|c} 
Courcing & Sole sourcing & Dual sourcing \\
\hline Non-cooperative & Scenario 1 & Scenario 2 \\
\hline Cooperative & Scenario 3 & Scenario 4 \\
\hline
\end{tabular}

Figure 1. Four sourcing scenarios obtained from combinations of sourcing and coordination strategies

\section{Figure 2}

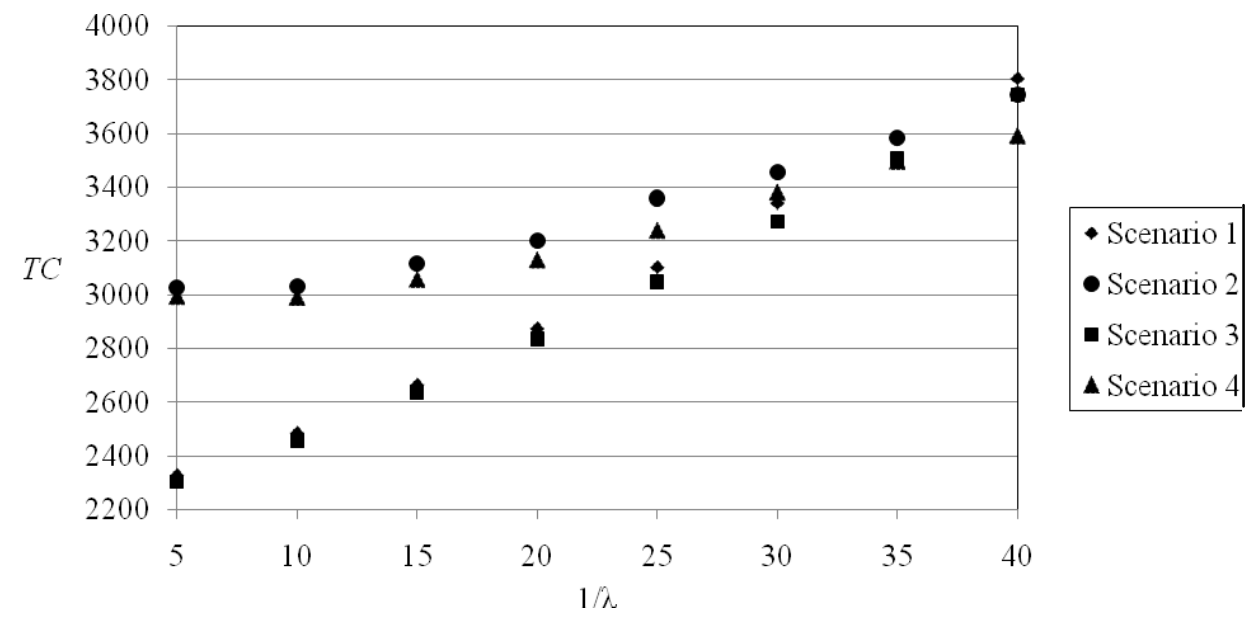

Figure 2. Effect of lead-time variation on scenario rankings

\section{Figure 3}

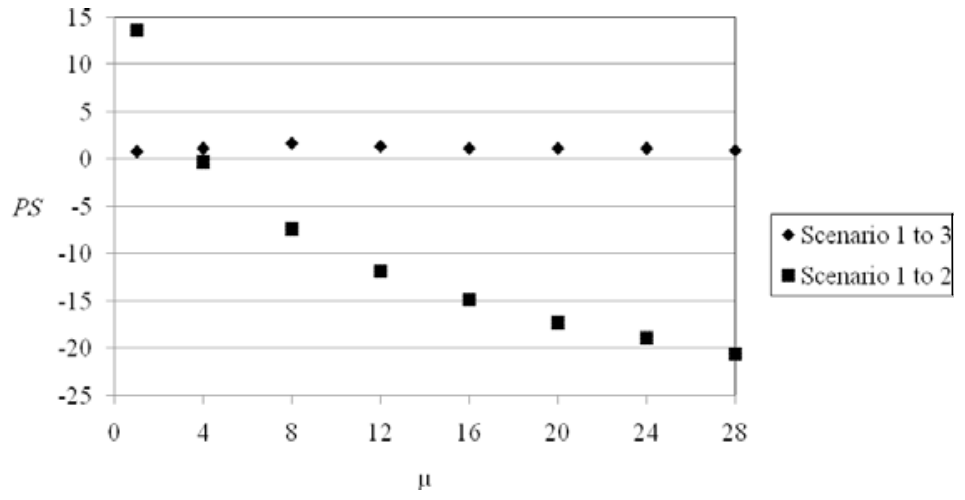

Figure 3. Effect of ordering and setup costs on scenario shifts 


\section{Figure 4}

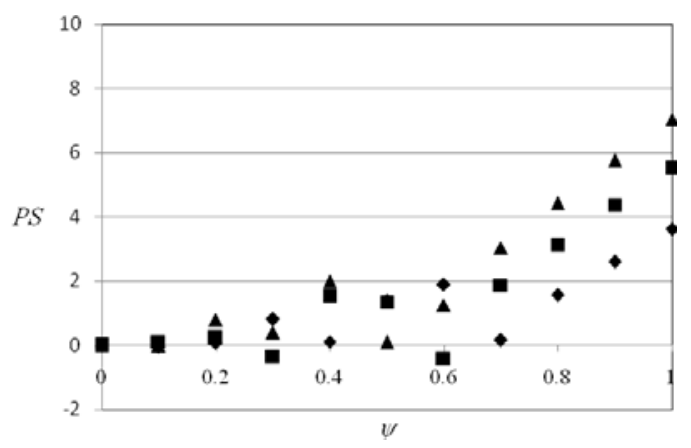

(a) Difference between savings percentages for a shift to the dual-sourcing strategy under cooperative and non-cooperative strategies

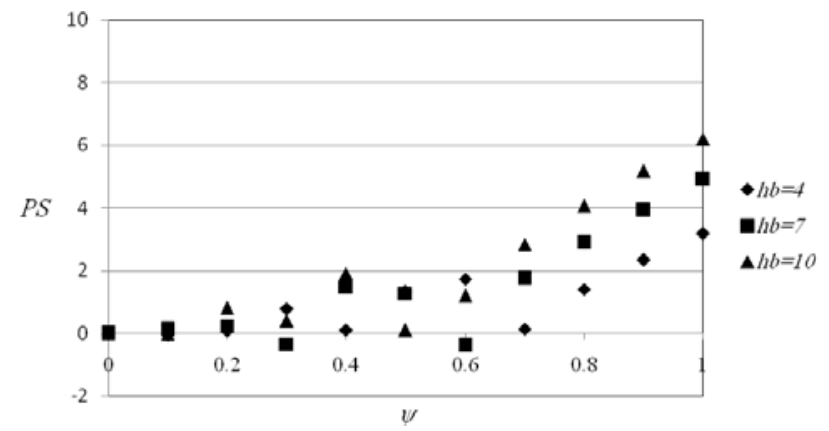

(b) Difference between savings percentages for a shift to the cooperative strategy under sole and dual-sourcing strategies

Figure 4. Effect of $\psi$ on scenario shifts

\section{Figure 5}

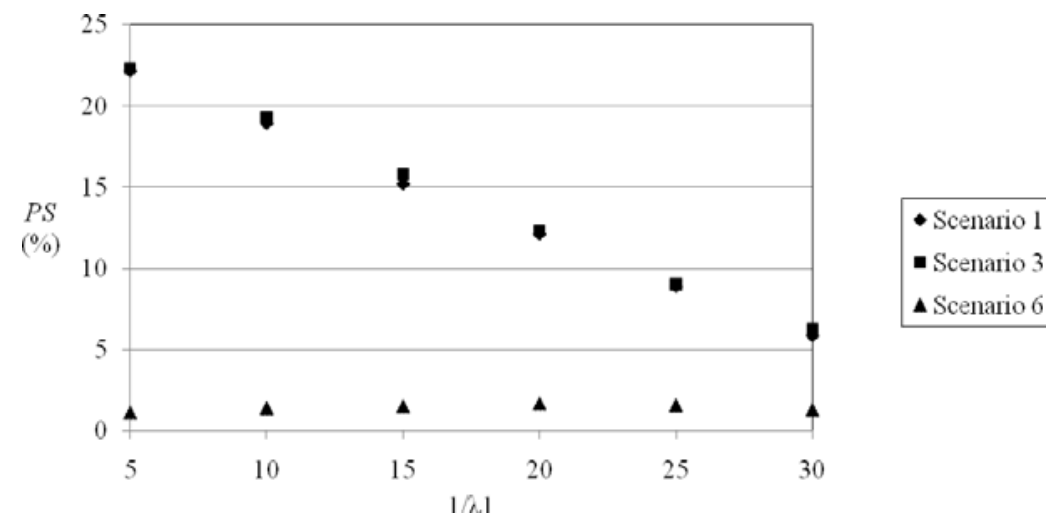

Figure 5. Effect of lead-time variation on percentage cost savings relative to Scenario 5

\section{Figure 6}

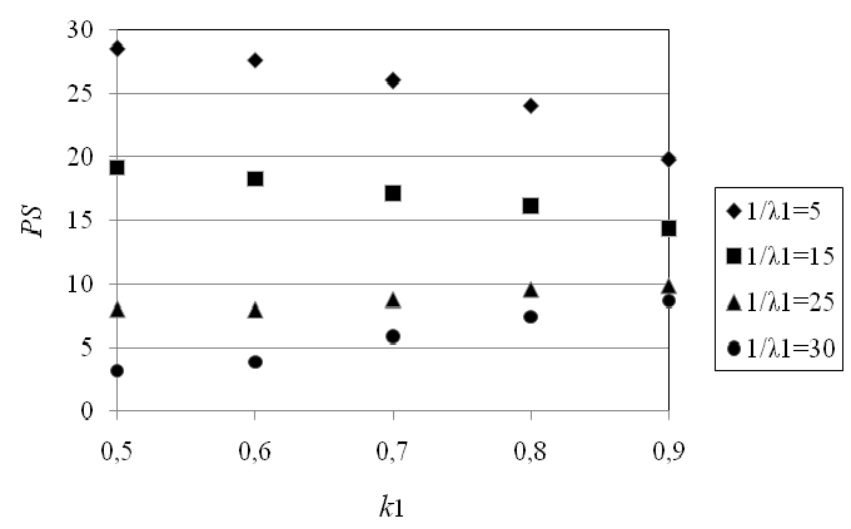

Figure 6. Effect of order split proportion $k_{1}$ on percentage cost savings when shifting

from Scenario 6 to Scenario 3 
Figure 7

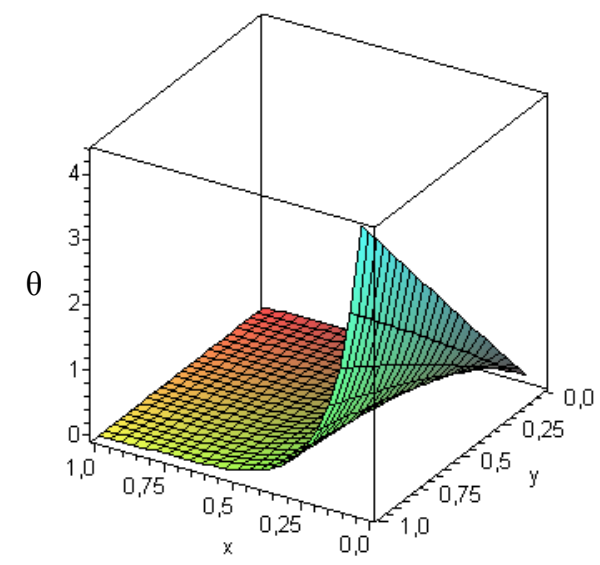

Figure 7. Value of $\theta$ for the full ranges of parameters $x$ and $y$ 


\section{Working Papers from CORAL - Centre for Operations Research Applications in Logistics}

L-2009-02 Mohsen S. Sajadieh, Anders Thorstenson \& Mohammad R. Akbari Jokar: Comparing sourcing strategies in two-echelon supply chains.

L-2009-01 Mohsen S. Sajadieh, Anders Thorstenson \& Mohammad R. Akbari Jokar: An integrated vendor-buyer model with stock-dependent demand.

L-2008-09 Ellis L. Johnson \& Sanne Wøhlk: Solving the Capacitated Arc Routing Problem with Time Windows using Column Generation.

L-2008-08 Søren Bloch \& Christian H. Christiansen: Simultaneously Optimizing Storage Location Assignment at Forward Area and Reserve Area - a Decomposition Based Heuristic.

L-2008-07 Bisheng Du \& Christian Larsen: Base stock policies with degraded service to larger orders.

L-2008-06 Karina H. Kjeldsen: Classification of routing and scheduling problems in liner shipping.

L-2008-05 Daniele Pretolani, Lars Relund Nielsen, Kim Allan Andersen \& Matthias Ehrgott: Time-adaptive versus history-adaptive strategies for multicriterion routing in stochastic time-dependent networks.

L-2008-04 Christian Larsen: Comments to Özkaya et al. (2006).

L-2008-03 Christian Larsen: Derivation of confidence intervals of service measures in a base-stock inventory control system with low-frequent demand.

L-2008-02 Jens Lysgaard: The Pyramidal Capacitated Vehicle Routing Problem.

L-2008-01 Jens Lysgaard \& Janni Løber: Scheduling participants of Assessment Centres.

L-2007-03 Christian Larsen: Note: Comments on the paper by Rosling (2002).

L-2007-02 Christian Larsen, Claus Hoe Seiding, Christian Teller \& Anders Thorstenson: An inventory control project in a major Danish company using compound renewal demand models.

L-2007-01 Christian Larsen: The $Q(s, S)$ control policy for the joint replenishment problem extended to the case of correlation among item-demands.

L-2006-11 Daniele Pretolani, Lars Relund Nielsen \& Kim Allan Andersen: A note on "Multicriteria adaptive paths in stochastic, time-varying networks".

L-2006-10 Lars Relund Nielsen, Kim Allan Andersen \& Daniele Pretolani: Bicriterion a priori route choice in stochastic time-dependent networks. 
L-2006-09 Christian Larsen \& Gudrun P. Kiesmüller: Developing a closed-form cost expression for an $(R, s, n Q)$ policy where the demand process is compound generalized Erlang.

L-2006-08 Eduardo Uchoa, Ricardo Fukasawa, Jens Lysgaard, Artur Pessoa, Marcus Poggi de Aragão, Diogo Andrade: Robust Branch-Cut-and-Price for the Capacitated Minimum Spanning Tree Problem over a Large Extended Formulation.

L-2006-07 Geir Brønmo, Bjørn Nygreen \& Jens Lysgaard: Column generation approaches to ship scheduling with flexible cargo sizes.

L-2006-06 Adam N. Letchford, Jens Lysgaard \& Richard W. Eglese: A Branch-andCut Algorithm for the Capacitated Open Vehicle Routing Problem.

L-2006-05 Ole Mortensen \& Olga W. Lemoine: Business integration between manufacturing and transport-logistics firms.

L-2006-04 Christian H. Christiansen \& Jens Lysgaard: A column generation approach to the capacitated vehicle routing problem with stochastic demands.

L-2006-03 Christian Larsen: Computation of order and volume fill rates for a base stock inventory control system with heterogeneous demand to investigate which customer class gets the best service.

L-2006-02 Søren Glud Johansen \& Anders Thorstenson: Note: Optimal base-stock policy for the inventory system with periodic review, backorders and sequential lead times.

L-2006-01 Christian Larsen \& Anders Thorstenson: A comparison between the order and the volume fill rates for a base-stock inventory control system under a compound renewal demand process.

L-2005-02 Michael M. Sørensen: Polyhedral computations for the simple graph partitioning problem.

L-2005-01 Ole Mortensen: Transportkoncepter og IT-støtte: et undersøgelsesoplæg og nogle foreløbige resultater.

L-2004-05 Lars Relund Nielsen, Daniele Pretolani \& Kim Allan Andersen: $K$ shortest paths in stochastic time-dependent networks.

L-2004-04 Lars Relund Nielsen, Daniele Pretolani \& Kim Allan Andersen: Finding the $K$ shortest hyperpaths using reoptimization.

L-2004-03 Søren Glud Johansen \& Anders Thorstenson: The $(r, q)$ policy for the lostsales inventory system when more than one order may be outstanding. 
L-2004-02 Erland Hejn Nielsen: Streams of events and performance of queuing systems: The basic anatomy of arrival/departure processes, when focus is set on autocorrelation.

L-2004-01 Jens Lysgaard: Reachability cuts for the vehicle routing problem with time windows. 
ISBN 9788778824332

Department of Business Studies

Aarhus School of Business

Aarhus University

Fuglesangs Allé 4

DK-8210 Aarhus V - Denmark

Tel. +4589486688

Fax +4586150188

www.asb.dk 